\title{
A phase II multicenter trial of concurrent chemoradiotherapy with weekly nedaplatin in advanced uterine cervical carcinoma: Tohoku Gynecologic Cancer Unit Study
}

\author{
YOSHIHITO YOKOYAMA ${ }^{1}$, TADAO TAKANO ${ }^{2}$, KENJI NAKAHARA $^{3}$, TADAHIRO SHOJI $^{4}$, HIROKAZU SATO $^{5}$, \\ HIDEKAZU YAMADA ${ }^{6}$, NOBUO YAEGASHI ${ }^{2}$, KUNIHIRO OKAMURA ${ }^{2}$, HIROHISA KURACHI ${ }^{3}$, \\ TORU SUGIYAMA ${ }^{4}$, TOSHINOBU TANAKA ${ }^{5}$, AKIRA SATO ${ }^{6}$, TORU TASE ${ }^{7}$ and HIDEKI MIZUNUMA ${ }^{1}$ \\ ${ }^{1}$ Department of Obstetrics and Gynecology, Hirosaki University School of Medicine, 5 Zaifu-cho, Hirosaki 036-8562; \\ ${ }^{2}$ Department of Obstetrics and Gynecology, Tohoku University School of Medicine, 1-1 Seiryo-cho, Aoba-ku, \\ Sendai 980-8574, Sendai; ${ }^{3}$ Department of Obstetrics and Gynecology, Yamagata University School of Medicine, \\ 2-2-2 Iidanishi, Yamagata 990-9585; ${ }^{4}$ Department of Obstetrics and Gynecology, Iwate Medical University \\ School of Medicine, 19-1 Uchimaru, Morioka 020-8505; ${ }^{5}$ Department of Obstetrics and Gynecology, \\ Akita University School of Medicine, 1-1-1 Hondo, Akita 010-8543; ${ }^{6}$ Department of Obstetrics and \\ Gynecology, School of Medicine Fukushima Medical University, 1 Hikarigaoka, Fukushima 960-1295; \\ ${ }^{7}$ Department of Gynecology, Miyagi Cancer Center, 47-1 Aishima, Natori 981-1293, Japan
}

Received November 6, 2007; Accepted January 29, 2008

\begin{abstract}
The purpose of this study was to evaluate the effectiveness and safety of concurrent chemoradiotherapy using weekly nedaplatin for the treatment of locally advanced squamous cell carcinoma of the uterine cervix. Nedaplatin at $30 \mathrm{mg} / \mathrm{m}^{2}$ was administered weekly 6 times with a concurrent external beam and intracavity radiotherapy. External beam radiation was delivered with a fraction dose of 2 Gy per day for 5 days a week during a 5-week period and intracavitary brachytherapy, of which the fraction size is 6 Gy to point A, was given once a week for a total of 4 times using a remote after-loading system. Forty-five patients were enrolled in this trial between April 2003 and December 2006. Of the 45 patients, $40(88.9 \%)$ completed the scheduled treatment and were evaluated for efficacy and safety. Of these, 4 were stage $\mathrm{Ib} 2,12$ were stage IIb, 18 were stage IIIb and 6 were stage IVa. The age distribution ranged from 27 to 79 years with a median age of 58 . The 40 patients achieved an objective response, $36(90 \%)$ a complete response and $4(10 \%)$ a partial response. At a median follow-up of 29 months (range, 8-52), the 3-year progression-free and overall survival were $58.7 \%$ (95\% confidence interval, $42-75 \%)$ and $78.0 \%(95 \%$
\end{abstract}

Correspondence to: Dr Yoshihito Yokoyama, Department of Obstetrics and Gynecology, Hirosaki University School of Medicine, 5 Zaifu-cho, Hirosaki 036-8562, Japan

E-mail: yokoyama@cc.hirosaki-u.ac.jp

Key words: advanced uterine cervical carcinoma, weekly nedaplatin, radiotherapy, phase II multicenter trial confidence interval, 56-90.0\%), respectively. Acute toxicities were transient and rendered non-lethal. Of the 45 patients enrolled for the trial, only $3(6.7 \%)$ had grade 4 leukopenia and neutropenia, respectively. Grade 3 diarrhea and nausea/ vomiting were observed in $2(4.4 \%)$ and $1(2.2 \%)$, respectively. These results indicate that weekly nedaplatin of $30 \mathrm{mg} / \mathrm{m}^{2}$ with concurrent radiotherapy is an effective and well-tolerated regimen for advanced squamous cell carcinoma of the uterine cervix.

\section{Introduction}

Although uterine cervical carcinomas have long been treated with surgery, radiation therapy alone, or a combination of the two, these treatments have a poor outcome, a fact resulting in a long-term debate on the need to combine them with chemotherapy. In the 1990s, numerous attempts were made to improve the prognosis for advanced uterine cervical carcinoma by concurrently using radiation therapy and chemotherapy. These attempts led to the announcement by the National Cancer Institute in the USA in February 1999 that the results of five randomized controlled trials demonstrated that the concurrent use of radiotherapy and chemotherapy (especially using cisplatin) was effective against advanced uterine cervical carcinoma and decreased the risk of death thereof by $30-50 \%$ (1-5). This concurrent chemoradiotherapy (CCRT) has become a current standard treatment in the USA for locally advanced uterine cervical carcinoma or pelvic extension of the carcinoma. However, data from these five clinical trials also showed several problems when this CCRT is employed in Japanese patients. The problems that need to be solved include that standard radiation therapy in Western countries is different to that in Japan. For example, most of the institutions 
in Japan use the high-dose-rate remote after-loading system (HDR-RALS) for intracavitary brachytherapy as the standard treatment modality for uterine cervical carcinoma; whether or not the reported dose of cisplatin is appropriate for Japanese women, cisplatin should be administered weekly or at a longer interval than that, the use of cisplatin is appropriate for patients with advanced uterine cervical carcinoma because of their possibly reduced renal function and how effective platinumcontaining drugs in the place of cisplatin will be in such patients, as well as how severe the late effects will be after the use of cisplatin.

Nedaplatin, an antineoplastic drug containing a platinum complex, was developed in order to provide superior antitumor effects to cisplatin and fewer adverse reactions such as the renal and gastrointestinal toxicities. Based on the results of a phase I clinical trial of nedaplatin, it was determined that the drug should be given as an intravenous infusion of $100 \mathrm{mg} / \mathrm{m}^{2}$ at intervals of four weeks (6). A phase II clinical trial using this dosage regimen demonstrated a response rate of $46.3 \%$ (19/41 patients) in patients with uterine cervical carcinoma (7), which was superior to that obtained with cisplatin $(35.9 \%$, $14 / 39$ patients). Regarding adverse drug reactions, although it was confirmed that the nephrotoxicity of nedaplatin was milder than that of cisplatin, certain patients developed grade 3 or 4 myelosuppression (33.6\% for thrombocytopenia and $31.1 \%$ for leukopenia), which suggested that the use of nedaplatin needs extreme caution (7).

Since nedaplatin is associated with a higher response rate in uterine cervical carcinoma than in cisplatin, fewer gastrointestinal and renal disorders, and less fluid volume are needed. The replacement of cisplatin with nedaplatin may therefore be expected to provide a longer survival associated with a better QOL. Yoshinaga et al conducted a dose-finding study of CCRT using weekly nedaplatin in uterine cervical carcinoma and confirmed that the recommended dose is $35 \mathrm{mg} / \mathrm{m}^{2}$ (8). Two phase I studies of radiation therapy combined with nedaplatin showed the optimal dose of weekly nedaplatin to be $30 \mathrm{mg} / \mathrm{m}^{2}$, which could be administered with minor adverse drug reactions and with no delayed radiation therapy or postponed surgery plan $(9,10)$.

At present, no evidence of CCRT in Japanese patients with advanced uterine cervical carcinoma exists. In our study, a multicenter trial of CCRT was planned using weekly nedaplatin in uterine cervical carcinoma to evaluate its tumor reduction effects, duration of response, survival time and occurrence of adverse events.

\section{Patients and methods}

Patients. Eligibility criteria were: 1) radiotherapy chosen as an initial treatment of the uterine cervical carcinoma and pathologically-proven squamous cell carcinoma, 2) target lesion measurable with RECIST, 3) age 80 years or younger, 4) the Eastern Cooperative Oncology Group (ECOG) performance status (PS) 0 or 1 and 5) the adequate function of the bone marrow, kidney and liver (leukocyte count $>3000 / \mathrm{mm}^{3}$, neutrophil count $>1500 / \mathrm{mm}^{3}$, platelet count $>100,000 / \mathrm{mm}^{3}$, total bilirubin $<1.5 \mathrm{mg} / \mathrm{dl}$, GOT (AST) or GPT (ALT) level less than twice the upper normal limit, and serum creatinine less than the upper normal limit). Acute toxicity
Table I. Patient characteristics $(n=45)$.

\begin{tabular}{lr}
\hline Age (years) & 58 \\
Median & $27-29$ \\
Range & \\
FIGO stage & 4 \\
Ib2 & 14 \\
IIb & 21 \\
IIIb & 6 \\
IVa & \\
Tumor size (cm) & 6 \\
$<4$ & 17 \\
$4-6$ & 19 \\
$6-8$ & 3 \\
$>8$ & \\
Pelvic lymphadenopathy & 20 \\
Yes & 25 \\
No & \\
\hline
\end{tabular}

was assessed according to the National Cancer Institute Common Toxicity Criteria, version 2.0, issued in 1999. Women were excluded from this study if they met any of the following criteria: 1) receiving prior radiotherapy or systemic chemotherapy for other diseases, 2) having other active carcinomas, 3) having serious complications or medical contraindications to chemotherapy and 4) showing disease outside the pelvic area or spread to the para-aortic lymph nodes. The study protocol was approved by the ethics committee of the Institutional Review Board of each participant institution. All of the patients gave their written informed consent before entering the trial. Registration of the patients was started in April 2003 and ended in December 2006.

Chemotherapy. Nedaplatin $\left(30 \mathrm{mg} / \mathrm{m}^{2}\right)$ was dissolved in $500 \mathrm{ml}$ saline solution and infused intravenously for $>180 \mathrm{~min}$. The first administration was performed on the starting day of the external beam radiation therapy. The regimen was repeated weekly for a total of six times. Nedaplatin infusion was completed $1 \mathrm{~h}$ before irradiation. Anti-emetics such as the 5-HT3 receptor antagonist were administered routinely before nedaplatin infusion. A granulocyte colony-stimulating factor was injected subcutaneously if patients had a neutrophil count of grade $\geq 3$. Nedaplatin infusion was delayed if the peripheral neutrophil count was $<1000 / \mathrm{mm}^{3}$ or the peripheral platelet count was $<75,000 / \mathrm{mm}^{3}$.

Radiotherapy. External beam radiation was delivered with anteroposterior and posteroanterior opposed beams generated by an X-ray accelerator with an energy of $10 \mathrm{MV}$ at a distance of $100 \mathrm{~cm}$. Whole pelvis irradiation to a total dose of $50 \mathrm{~Gy}$ was delivered with a fraction dose of 2 Gy per day for 5 days a week during a 5-week period. After $\sim 30$ Gy was given, a central shield with a width of $4 \mathrm{~cm}$ at the midline was used and intracavitary brachytherapy was performed using a RALS with a Co-60 source. Intracavitary brachytherapy, of which 
Table II. Response to the treatment.

\begin{tabular}{|c|c|c|c|c|c|}
\hline & $\begin{array}{c}\text { No. of } \\
\text { patients }\end{array}$ & CR & PR & SD & PD \\
\hline All stages & 40 & $36(90.0)$ & $4(10.0)$ & $0(0)$ & $0(0)$ \\
\hline Stage Ib2-IIb & 16 & $15(93.8)$ & $1(6.20)$ & $0(0)$ & $0(0)$ \\
\hline Stage IIIb-IVa & 24 & $21(87.5)$ & $3(12.5)$ & $0(0)$ & $0(0)$ \\
\hline
\end{tabular}

$\mathrm{CR}$, complete response; PR, partial response; $\mathrm{SD}$, stable disease and PD, progressive disease.

Table III. Site of disease progression.

\begin{tabular}{lc}
\hline Site of progression & No. of patients $(\%)$ \\
\hline Local & $10(25)$ \\
Distant & $4(10)$ \\
No evidence of disease & $26(65)$ \\
\hline
\end{tabular}

Recurrences were classified as local if they were first detected in the pelvis, cervix or vagina and as distant if they were first detected outside the pelvis.

the fraction size is 6 Gy to point A, was given once a week for a total of 4 times. Radiotherapy was suspended when the peripheral neutrophil count was $<500 / \mathrm{mm}^{3}$ or the peripheral platelet count was $<50,000 / \mathrm{mm}^{3}$.

Patient evaluation. All the patients had weekly hematology and chemistry testing for safety as well as for the clinical evaluation of the disease during treatment. Patients were evaluated for response 4 weeks after finishing the treatment. Complete response was defined histologically and clinically as the disappearance of all gross lesions for 1 month after completion of the treatment. Partial response was defined as a $>50 \%$ reduction of the tumor size for 1 month after completion of the treatment and an absence of new lesions. Stable disease was defined as the presence of the tumor with $<50 \%$ reduction of the tumor size. Progressive disease was defined as the appearance of any new lesion during the treatment or a $>25 \%$ increase in size of the local tumor.

Statistical analysis. The progression-free survival (PFS) and overall survival (OS) were calculated from the end of the treatment by the Kaplan-Meier method. A comparison between the survival curves was made using the log-rank test. A difference in the response rate between stages was analyzed using the Chi-square test. Statistical significance was set at $\mathrm{P}<0.05$.

\section{Results}

Patient outcome. Forty-five patients were enrolled in this trial and were staged according to the International Federation of Gynecology and Obstetrics (FIGO) criteria. The number of patients with FIGO stage Ib2, IIb, IIIb and IVa was 4, 14, 21 and 6, respectively. Each of the 4 cases of FIGO stage Ib2 had a tumor diameter of $6 \mathrm{~cm}$ or larger. The age distribution ranged from 27 to 79 years with a median age of 58. The characteristics of these patients are listed in Table I. Forty patients out of the $45(88.9 \%)$ completed the trial and were evaluated for the efficacy and safety of this treatment. Of the 40 patients, 33 were treated with external beam radiation and intracavitary brachytherapy. The remaining 7 patients received only external beam radiation, the dose ranging from 50 to $60 \mathrm{~Gy}$. Out of the 45 enrolled patients, 5 abandoned the scheduled treatment. They voluntarily withdrew from the trial on the grounds of bone marrow suppression and anorexia. While all of the 45 enrolled in this trial were evaluated for toxicity, the 40 patients who completed the scheduled treatment were evaluated for response.

Patients who completed the planned treatment (36 out of the $40,90 \%$ ) showed a complete response and 4 out of those (10\%) showed a partial response, achieving a highly successful treatment $(100 \%)$ (Table II). No significant difference in the response rate between stages Ib2/IIb and IIIb/IVa (Table II) was found.

Fourteen patients relapsed or progressed after the treatment (Table III). Eleven complete responders relapsed (7 inside the radiation field, 3 in the para-aortic lymph nodes and 1 in the lungs). Of the 4 partial responders, 1 underwent a radical hysterectomy shortly after the completion of treatment and she survived without any evidence of disease for 43 months. The remaining 3 partial responders progressed locally despite palliative treatment.

At a median follow-up of 29 months (range, 8-52), the 3 -year PFS and OS rates were $58.7 \%$ (95\% confidence interval, $42-75 \%$ ) and $78.0 \%$ (95\% confidence interval, 56-90.0\%), respectively (Fig. 1a and b). The 3-year PFS and OS rates were 68.7 and $86.5 \%$ for patients with stage Ib2 and IIb, respectively and 47.9 and $60.4 \%$ for those with stage IIIb and IVa, respectively. However, these differences were not significant (Table IV). The 3-year PFS and OS rates were significantly lower for the patients with pelvic lymph node swelling than for those without $(74.5$ vs. $39.2 \%, \mathrm{P}<0.01$ and 89.3 vs. $41.1 \%, \mathrm{P}<0.02$, respectively) (Table IV). No significant differences in the 3 -year PFS and OS were seen between the tumor sizes (Table IV).

Toxicity. Acute toxicities were transient and rendered nonlethal. Of the 45 patients enrolled in the trial, only $3(6.7 \%)$ had grade 4 leukopenia and neutropenia, respectively (Table V). No patient required blood or platelet transfusions. Grade 3 
Table IV. The 3-year PFS and OS rates according to the clinical factors.

\begin{tabular}{|c|c|c|c|c|c|}
\hline & No. of patients & The 3-year PFS (\%) & P-value & The 3-year OS (\%) & P-value \\
\hline \multicolumn{6}{|l|}{ Stage } \\
\hline $\mathrm{Ib} 2 / \mathrm{IIb}$ & 16 & 68.7 & & 86.5 & \\
\hline IIIb/IVa & 24 & 47.9 & 0.380 & 60.4 & 0.160 \\
\hline \multicolumn{6}{|c|}{ Tumor size $(\mathrm{cm})$} \\
\hline$\leq 6$ & 19 & 52.3 & & 69.4 & \\
\hline$>6$ & 21 & 64.3 & 0.450 & 76.9 & 0.630 \\
\hline \multicolumn{6}{|c|}{ Pelvic lymph nodes } \\
\hline Swelling (-) & 23 & 74.4 & & 89.3 & \\
\hline Swelling (+) & 17 & 39.2 & 0.010 & 41.1 & 0.016 \\
\hline
\end{tabular}

PFS, progression-free survival and OS, overall survival.
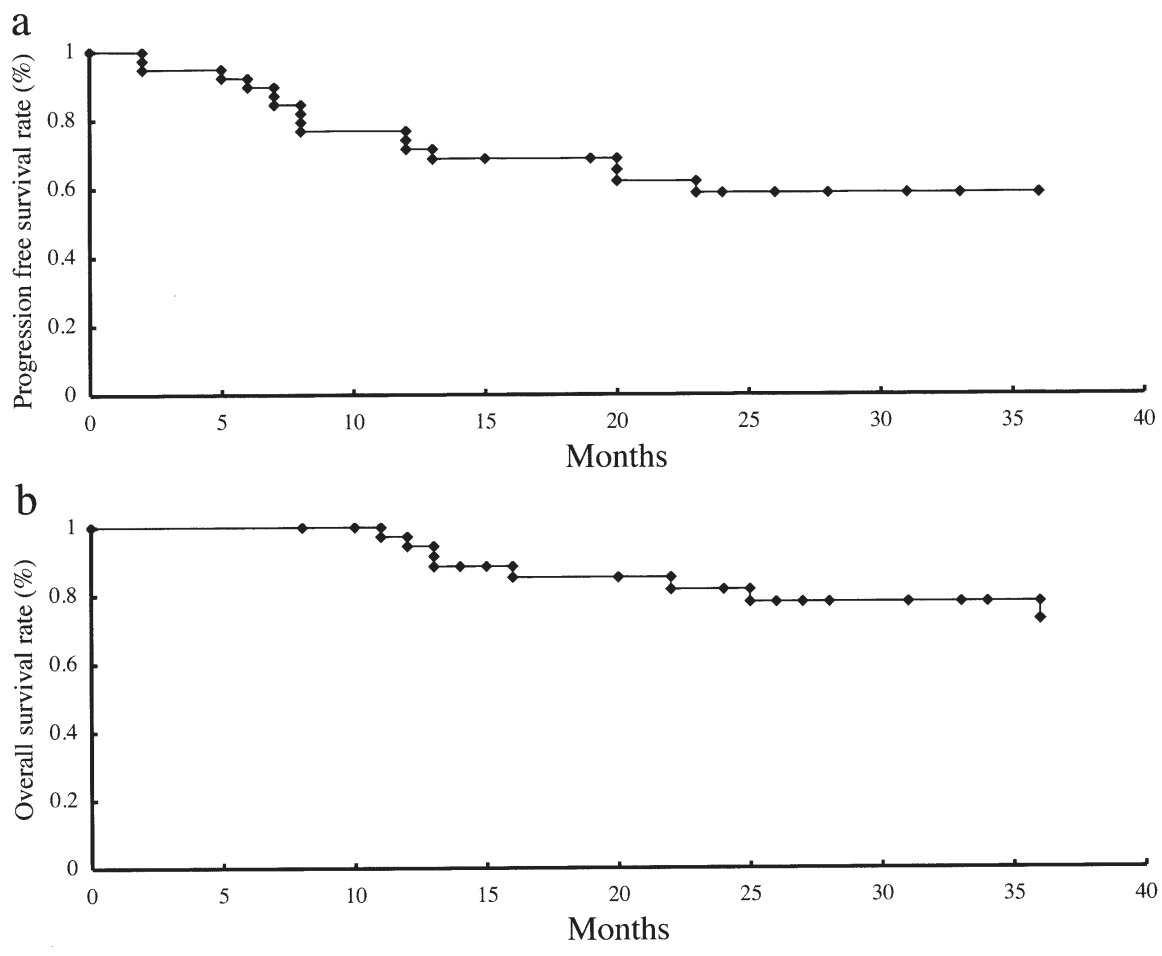

Figure 1. The 3-year progression-free survival at a median follow-up of 29 months (range, 8-52) (a) and the 3-year overall survival at a median follow-up of 29 months (range, 8-52) (b).

diarrhea and nausea/vomiting were observed in $2(4.4 \%)$ and 1 $(2.2 \%)$, respectively (Table V). The chemotherapy was delayed for a week due to adverse reactions in 5 out of the 40 patients who completed the treatment. There were no patients in whom the chemotherapy was delayed for 2 weeks or longer. The radiation therapy was suspended for 4-5 days at the same time in 4 out of the 5 patients who delayed the chemotherapy. One patient suffered from radiation proctitis as a delayed adverse reaction and received surgical treatment (Table V). She survived without any evidence of disease.

\section{Discussion}

In this phase II trial, we achieved a $90 \%$ complete response rate, and all the patients had a successful response. The 3-year
PFS and OS rates were 58.7 and $78.0 \%$, respectively (Fig. 1a and b). In addition, $~ 90 \%$ of the enrolled patients completed the trial with a few grade 4 hematological toxicities (6.7\%), indicating that weekly nedaplatin of $30 \mathrm{mg} / \mathrm{m}^{2}$ with concurrent radiotherapy is an effective and well-tolerated regimen for advanced uterine cervical carcinoma.

In the phase II studies of cisplatin and concurrent radiotherapy for advanced uterine cervical carcinoma, Malfetano et al reported that $94 \%$ of the patients completely responded and $75 \%$ survived without evidence of disease with a mean follow-up of 47.5 months (11). However, their scheduled treatment was characterized by extended-field radiation therapy including the para-aortic lymph nodes. In contrast, Fields et al, reported that $87 \%$ of the patients with this disease were complete responders and the 5-year survival was $67 \%$ 
Table V. Toxicity profile (45 patients).

\begin{tabular}{lcc}
\hline Toxicity & Grade 3 $(\%)$ & Grade 4 (\%) \\
\hline Hematological & $17(37.8)$ & $3(6.7)$ \\
Leukopenia & $14(31.1)$ & $3(6.7)$ \\
Neutropenia & $3(6.7)$ & 0 \\
Anemia & $2(4.4)$ & 0 \\
Thrombocytopenia & & \\
Non-hematological & $2(4.4)$ & 0 \\
Diarrhea & $1(2.2)^{\mathrm{a}}$ & 0 \\
Proctitis & $1(2.2)$ & 0 \\
Nausea and vomiting &
\end{tabular}

${ }^{\mathrm{a} A c c o r d i n g}$ to the radiation therapy oncology group late-radiation morbidity-scoring scheme.

for stage III and $25 \%$ for stage IV with a median follow-up time of 65 months (12). The response rate of the present trial with nedaplatin and concurrent radiotherapy is comparable to that of the two previous trials with cisplatin and concurrent radio-therapy. However, while $60.0 \%$ of the patients in this trial were FIGO stages IIIb or IVa, only 34.3 and $50.9 \%$ were those stages in the two previous trials, respectively.

Five randomized phase III trials have an OS advantage for cisplatin-based chemotherapy with concurrent radiotherapy. Although these trials vary in terms of the stage of disease and schedule of cisplatin and radiation, all of them showed a significant survival benefit for the combined treatment, decreasing the risk of death by $30-50 \%$ (1-5). However, the local recurrence rate, which is between 19 and $24 \%$, is still high. Similarly, the local recurrence rate in this trial was $25 \%$.

As shown in Table IV, the pelvic lymph node status significantly influenced the 3-year PFS and OS rates. Of the 4 patients who had distant recurrence, 3 relapsed in the paraaortic lymph node (PAN) region (Table III). Of the 3 patients with PAN metastasis, 2 also had a pelvic lymphadenopathy. Regarding the independent factor influencing the OS rate for the patients who had a high-risk uterine cervical carcinoma and were treated with intra-arterial cisplatin/nedaplatin, intravenous 5-fluorouracil and concurrent radiotherapy, Kawase et al reported that the pelvic lymph node status was the only risk factor and recommended that patients without a pelvic lymphadenopathy should be selected as candidates for CCRT (13). It is suggested that the drug concentration in the pelvic lymph nodes was low in patients with a pelvic lymphadenopathy and these patients were reported to respond poorly to CCRT (13).

In this study, the para-aortic recurrence was $7.5 \%$ and was compatible with the results reported by Eifel et al who showed that the rate of para-aortic recurrence in advanced uterine carcinoma patients treated with pelvic radiotherapy, concurrently using fluorouracil and cisplatin, was 7\% at 5 years (14). In addition, Eifel et al showed that pelvic radiation with concurrent chemotherapy significantly prolonged the OS of advanced uterine cervical carcinoma patients without PAN involvement, compared to extended-field radiation including para-aortic radiation, suggesting that prophylactic para-aortic radiation does not appear to be appropriate for the treatment of locally advanced cervical carcinoma (14). However, Malfetano et al suggested the effectiveness of para-aortic radiation in combination with chemotherapy. They showed that although $7(10.7 \%)$ out of the 67 locally advanced cervical carcinoma patients treated with extended-field radiation, including prophylactic para-aortic radiation and weekly cisplatin had a distant failure, no patient had PAN failure (11). Thus, it is still uncertain whether the addition of prophylactic para-aortic radiation to CCRT may improve the therapeutic effect on nodal involvement and prevent nodal failure, which warrants further investigation for the improvement of survival of patients with a pelvic lymphadenopathy.

In this phase II trial, grade 3 and 4 leukopenia was found in 37.8 and $6.7 \%$ of the enrolled patients, respectively but no grade 4 non-hematological toxicity (Table V). Of the 45 enrolled patients, $5(11.1 \%)$ voluntarily withdrew from the trial due to bone marrow suppression and anorexia, although the levels of the adverse reactions were medically mild. In this trial, nedaplatin was administered at a total dosage of $180 \mathrm{mg} / \mathrm{m}^{2}$ to each patient. The completion rate was $\sim 90 \%$ and the delayed duration was a maximum of 1 week, suggesting that the present regimen of using weekly nedaplatin and radiotherapy was clinically effective and welltolerated.

The results of the GOG protocols 120 and 123 after using radiation and concurrent cisplatin demonstrated that the incidence of grade 3-4 leukopenia was 13.1 and $21.3 \%$, respectively $(2,3)$. In these studies, the dose of cisplatin administered to each subject was 192 and $143 \mathrm{mg} / \mathrm{m}^{2}$ or more at the GOG protocols 120 and 123 , respectively $(2,3)$. However, Ohno et al, who employed a dose of cisplatin at either 100 or $151 \mathrm{mg} / \mathrm{m}^{2}$ with concurrent radiation, reported that the incidence of grade 3-4 leukopenia was 50 and 58\%, respectively (15). Moreover, Mitsuhashi et al reported that the incidence of grade 3-4 leukopenia treated with cisplatin and concurrent radiation reached $60 \%$ at the dosage of $168 \mathrm{mg} / \mathrm{m}^{2}$ of cisplatin per patient (16). These differences in the incidence of adverse reactions suggest that the tolerance for cisplatin in Japanese women may be lower than that in American women. It is suggested that cisplatin is a radiation sensitizer with the ability to inhibit the repair of sublethal radiation damage. Fu et al and Dewit showed an enhancement of cell kill in various tumors by the concomitant administration of cisplatin and radiation $(17,18)$. Nedaplatin is also reported to have radiation-sensitizing properties but its exact mechanism has yet to be understood. Nakamura et al showed that although the antitumor effect of nedaplatin on xenografted human brain tumors was comparable to that after exposure to the radiation, the peak incidence of apoptosis in the tumor after treatment with nedaplatin was much lower than that after exposure to the radiation and suggested that a different mechanism rather than the p53-dependent apoptosis be postulated for the antineoplastic activity caused by nedaplatin (19). More recently, Tanaka et al reported that the antiproliferative effect of nedaplatin on uterine cervical carcinoma cells could be enhanced several hours before or after radiation, suggesting that nedaplatin may be the most appropriate anticancer drug for CCRT (20). 
This is the first report to evaluate the effectiveness and safety of nedaplatin and concurrent radiation in advanced uterine cervical carcinoma. Although the statistical power of this study is limited due to the small number of subjects, the results in this trial are sufficient to warrant further investigation. A randomized phase III trial with this or similar regimens should be conducted to validate whether nedaplatin is equal to or superior to cisplatin in the survival of patients with this disease.

\section{References}

1. Morris M, Eifel PJ, Lu J, Grigsby PW, Levenback C, Stevens RE, Rotman M, Gershenson DM and Mutch DG: Pelvic radiation with concurrent chemotherapy compared with pelvic and paraaortic radiation for high-risk cervical cancer. N Engl J Med 340: 1137-1143, 1999

2. Rose PG, Bundy BN, Watkins EB, Thigpen JT, Deppe G, Maiman MA, Clarke-Pearson DL and Insalaco S: Concurrent cisplatin-based radiotherapy and chemotherapy for locally advanced cervical cancer. N Engl J Med 340: 1144-1153, 1999.

3. Keys HM, Bundy BN, Stehman FB, Muderspach LI, Chafe WE, Suggs CL III, Walker JL and Gersell D: Cisplatin, radiation, and adjuvant hysterectomy compared with radiation and adjuvant hysterectomy for bulky stage IB cervical carcinoma. N Engl J Med 340: 1154-1161, 1999.

4. Whitney CW, Sause W, Bundy BN, Malfetano JH, Hannigan EV, Fowler WC Jr, Clarke-Pearson DL and Liao SY: Randomized comparison of fluorouracil plus cisplatin versus hydroxyurea as an adjunct to radiation therapy in stage IIB-IVA carcinoma of the cervix with negative para-aortic lymph nodes: a gynecologic oncology group and southwest oncology group study. J Clin Oncol 17: 1339-1348, 1999.

5. Peters WA III, Liu PY, Barrett RJ II, Stock RJ, Monk BJ, Berek JS, Souhami L, Grigsby P, Gordon W Jr and Alberts DS: Concurrent chemotherapy and pelvic radiation therapy compared with pelvic radiation therapy alone as adjuvant therapy after radical surgery in high-risk early-stage cancer of the cervix. J Clin Oncol 18: 1606-1613, 2000.

6. Ota K, Wakui A, Majima H, Niitani H, Inuyama Y, Ogawa M, Ariyoshi Y, Yoshida O, Taguchi T and Kimura I: Phase I study of a new platinum complex 254-S, cis-diammine (glycolato)platinum (II). Gan To Kagaku Ryoho 19: 855-861, 1992.

7. Kato T, Nishimura H, Yakushiji M, Noda K, Terashima Y, Takeuchi S, Takamizawa H, Suzuki M, Arai M and Ota M: Phase II study of 254-S (cis-diammine glycolato-platinum) for gynecological cancer. Gan To Kagaku Ryoho 19: 695-701, 1992.

8. Yoshinaga K, Niikura H, Ogawa Y, Nemoto K, Nagase S, Takano T, Ito K and Yaegashi N: Phase I trial of concurrent chemoradiation with weekly nedaplatin in patients with squamous cell carcinoma of the uterine cervix. Gynecol Oncol 104: 36-40, 2007.
9. Inuyama Y, Miyake H, Horiuchi M, Hayasaki K, Komiyama S and Ota K: An early phase II clinical study of cis-diammine glycolato-platinum, 254-S, for head and neck cancers. Gan To Kagaku Ryoho 19: 863-869, 1992.

10. Onishi Y, Nakamura T, Hatae M, Adachi S and Ogasawara T: Phase I study of weekly nedaplatin and radiation therapy for advanced cervical cancer. Proc ASCO 21: 111b, 2002.

11. Malfetano JH, Keys H, Cunningham MJ, Gibbons S and Ambros R: Extended-field radiation and cisplatin for stage IIB and IIIB cervical carcinoma. Gynecol Oncol 67: 203-207, 1997.

12. Fields AL, Anderson PS, Goldberg GL, Wadler S, Beitler J, Sood B and Runowicz CD: Mature results of a phase II trial of concomitant cisplatin/pelvic radiotherapy for locally advanced squamous cell carcinoma of the uterine cervix. Gynecol Oncol 61: 416-422, 1996.

13. Kawase S, Okuda T, Ikeda M, Ishihara S, Itoh Y, Yanagawa S and Ishigaki $\mathrm{T}$ : Intra-arterial cisplatin/nedaplatin and intravenous 5-fluorouracil with concurrent radiation therapy for patients with high-risk uterine cervical cancer. Gynecol Oncol 102: 493-499, 2004.

14. Eifel PJ, Winter K, Morris M, Levenback C, Grigsby PW, Cooper J, Rotman M, Gershenson D and Mutch DG: Pelvic irradiation with concurrent chemotherapy versus pelvic and para-aortic irradiation for high-risk cervical cancer: An update of radiation therapy oncology group trial (RTOG) 90-01. J Clin Oncol 22: 872-880, 2004.

15. Ohno T, Kato S, Wakamatsu M, Noda S, Murakami C, Nakamura $\mathrm{M}$ and Tsujii $\mathrm{H}$ : Incidence and temporal pattern of anorexia, diarrhea, weight loss and leukopenia in patients with cervical cancer treated with concurrent radiation therapy and weekly cisplatin: Comparison with radiation therapy alone. Gynecol Oncol 103: 94-99, 2006.

16. Mitsuhashi A, Uno T, Tanaka N, Suzuki K, Tate S, Yamazawa K, Matsui H, Yamamoto S, Ito H and Sekiya S: Phase I study of daily cisplatin and concurrent radiotherapy in patients with cervical carcinoma. Gynecol Oncol 96: 194-197, 2005.

17. Fu KK, Lam KN and Rayner PA: The influence of time sequence of cisplatin administration and continuous low dose rate irradiation (CLDRI) on their combined effects on a murine squamous cell carcinoma. Int J Radiat Oncol Biol Phys 11: 2119-2124, 1985.

18. Dewit L: Combined treatment of radiation and cis-diamminedichloroplatinum (II): A review of experimental and clinical data. Int J Radiat Oncol Biol Phys 13: 403-426, 1987.

19. Nakamura Y, Hasegawa M, Hayakawa K, Mtsuura M, Suzuki Y, Nasu S, Yamakawa M, Mitsuhashi N and Niibe H: Induction of p53-dependent apoptosis in vivo by nedaplatin and ionizing radiation. Oncol Rep 7: 261-265, 2000.

20. Tanaka T, Yukawa K and Umesaki N: Radiation reduces carboplatin sensitivity and enhances nedaplatin sensitivity in cervical squamous cell carcinoma in vitro. Eur J Gynaecol Oncol 28: 352-355, 2007. 